\title{
Palmieri and Vesel on Symmetry and Harmony in Copernicus' Cosmology
}

\author{
Bernard R. Goldstein \\ University of Pittsburgh \\ brg@pitt.edu
}

\author{
Giora Hon \\ University of Haifa \\ hon@research.haifa.ac.il
}

In his review [Aestimatio 11 (2014) 188-190] of Matjaž Vesel's book, ${ }^{1}$ Paolo Palmieri focuses on the question 'What does Vesel mean by "Platonism" and can it be neatly defined in the context of European culture of the 16th century?' According to Vesel, as reported by Palmieri, harmonia and symmetria are two key concepts whose applications in De revolutionibus (1543) show Copernicus to be a follower of Plato. In this brief note, we seek to clarify Copernicus' explicit invocations of symmetry and harmony in his De revolutionibus.

Palmieri paraphrases part of the author's argument:

His [Copernicus'] central argument for the Earth's motion is, therefore, the firm symmetria of the universe, that is, the commensurability of its parts....

It is true that 'symmetria' in Euclid's Elements means commensurability but the context of Copernicus' usages indicates that the references in De revolutionibus are to the Vitruvian sense of well-proportioned as an aesthetic category rather than to a mathematical category [Hon and Goldstein 2004]. Both meanings of 'symmetria' are well attested from Antiquity to the 16th century — and even later. ${ }^{2}$

1 M. Vesel, Copernicus: Platonist Astronomer-Philosopher. Cosmic Order, the Movement of the Earth, and the Scientific Revolution. Frankfurt am Main: Peter Lang, 2015.

2 Indeed, both meanings of 'symmetria' can be found in the works of Plato: see Hon and Goldstein 2008, 70 and 94-95.

(C) 2014 Institute for Research in Classical Philosophy and Science All rights reserved
ISSN 1549-4497 (online) ISSN 1549-4470 (print) 
Copernicus considers Ptolemy's models a mixed bag of theories which together depict the universe as 'a monster'. In this tradition, astronomers could not

deduce from the eccentrics the principal consideration, that is, the structure of the universe and the true symmetry of its parts (ac partium eius certam symmetriam). On the contrary, their experience was just like someone taking from various places hands, feet, a head, and other parts of the body (membra), very well depicted indeed, but not for the representation of a single person; since these fragments would not belong to one another at all, a monster rather than a man would be put together from them. [Copernicus 1543, f. iii"; Rosen 1992, 4, slightly modified: cf. Hon and Goldstein 2008, 158]

When disparate elements are put together monstrosity, rather than a beautiful human form, is the result.

Copernicus then adds a reference to the world as a machine:

For a long time...I reflected on this confusion in the astronomical tradition concerning the derivation of the motions of the universe's spheres. I began to be annoyed that the movements of the world machine (motuum machinae mundi), created for our sake by the best and most orderly Artisan of all, were not understood with greater certainty by the philosophers.... [Copernicus 1543, f. iii ${ }^{\mathrm{v}}$; Rosen 1992, 4, slightly modified]

Despite the lack of an explicit reference to Vitruvius (first century Bc), we are persuaded that Copernicus expected the universe to comply with the Vitruvian notion of symmetry: a temple (the universe) ${ }^{3}$ whose constituent elements (the planetary orbs) relate to each other to form a beautiful whole, based on the order of their distances from the Sun (the center of motion for all of them). Vitruvius used the term symmetry to refer to the well-proportioned feature of the human body, the structure of a building, and the efficient functioning of a machine, treating separately these three domains in which symmetry is applied [De arch.3.1.2, 5.6.7, 10.10.1]. Copernicus invokes all three Vitruvian applications of symmetry.

At the juncture where Copernicus claims to have grasped the 'principal consideration', he asserts that

${ }^{3}$ For the universe as a most beautiful temple (in hoc pulcher[r]imo templo), see Copernicus, De rev. $1.10\left[1543,9^{v}\right]$. 
in this arrangement (sub hac ordinatione), therefore, we discover a marvelous symmetry of the universe (mundi symmetriam) and a truly harmonious linkage (harmoniae nexum) between the motion of the orbs and their size.... [De rev. 1.10 in $1543,10^{\mathrm{r}}$; Rosen 1992, 22, slightly modified]

Copernicus brings together two previously distinct aesthetic values: symmetry as proportionality in what is pleasing to the eye or efficient, and harmony as proportionality in what is pleasing to the ear. This harmony concerns the relation of the motions or, equivalently, the periods of the planetary orbs, where these orbs are ordered according to their distances from the center of motion, the Sun [cf. Hon and Goldstein 2008, 158-163]. In other words, the cosmos displays both symmetry and harmony. Copernicus thus takes advantage of the aesthetic value of both symmetry and harmony; for him, 'symmetry' does not have the general mathematical sense of 'commensurability'.

\section{BIBLIOGRAPHY}

Copernicus, N. 1543. De revolutionibus. Nuremberg.

Granger, F. 1957. ed. and trans. Vitruvius, On Architecture. 2 vols. Cambridge, MA/London, UK.

Hon, G. and Goldstein, B. R. 2004. 'Symmetry in Copernicus and Galileo'. Journal for the History of Astronomy 35:273-292.

2008. From Summetria to Symmetry: The Making of a Revolutionary Scientific Concept. Archimedes: New Studies in the History and Philosophy of Science and Technology 20. Dordrecht.

Rosen, E. 1992. trans. Nicholas Copernicus, On the Revolutions. Baltimore/ London. 